\title{
A TANGENTIAL CONVERGENCE FOR BOUNDED HARMONIC FUNCTIONS ON A RANK ONE SYMMETRIC SPACE
}

BY

\author{
JACEK CYGAN
}

\begin{abstract}
Let $u$ be a bounded harmonic function on a noncompact rank one symmetric space $M=G / K \approx N^{-} A, N^{-} A K$ being a fixed Iwasawa decomposition of $G$. We prove that if for an $a_{0} \in A$ there exists a $\operatorname{limit} \lim u\left(n a_{0}\right) \equiv c_{0}$, as $n \in N^{-}$goes to infinity, then for any $a \in A, \lim u(n a)=c_{0}$. For $M=$ $S U(n, 1) / S(U(n) \times U(1))=B^{n}$, the unit ball in $C^{n}$ with the Bergman metric, this is a result of Hulanicki and Ricci, and in this case it reads (via the Cayley transformation) as a theorem on convergence of a bounded harmonic function to a boundary value at a fixed boundary point, along appropriate, tangent to $\partial B^{n}$, surfaces.
\end{abstract}

0. Introduction. Let $M$ be a noncompact symmetric space of rank one. $M$ can be expressed as a homogeneous space $G / K$ where $G$ is a semisimple group of isometries of $M$ and $K$ is a maximal compact subgroup of $G$. Let $g, f$ denote the Lie algebras of $G$ and $K, B$ the Killing form of $\mathfrak{g}$, and $\mathfrak{p}$ the orthogonal complement of $f$ in $g$ relative to $B$. If $\pi: G \rightarrow G / K$ denotes the canonical projection, its differential at the identity, $\pi_{*}$, identifies the subspace $\mathfrak{p}$ of $g$ with $T_{0}(M)$, the tangent space of $M$ at the origin $o=\pi(e)$, and the invariant metric $g$ on $M$ can be chosen so that $g_{0}$ corresponds to the restriction of $B$ to $\mathfrak{p} \times \mathfrak{p}$ under the above identification. We denote by $\Delta$ the corresponding ( $G$-invariant) Laplace-Beltrami operator on $M$. A function $u \in C^{\infty}(M)$ is called harmonic if $\Delta u=0$. Let a be a maximal (one-dimensional) abelian subspace of $\mathfrak{p}, \alpha$ and possibly $2 \alpha$ in $a^{*}$, the corresponding system of positive restricted roots relative to the fixed choice of a "positive part" $a^{+}$in $a$. Let $g_{-\alpha}$ and $g_{-2 \alpha}$ denote the root spaces corresponding to $-\alpha$ and $-2 \alpha$. Then $\mathfrak{n}^{-}=\mathfrak{g}_{-\alpha} \oplus \mathfrak{g}_{-2 \alpha}$ is a nilpotent subalgebra of $\mathfrak{g}$ and one has the Iwasawa decomposition $G=N^{-} A K$, with $N^{-}=\exp \mathfrak{n}^{-}, A=\exp$ a. The above decomposition shows that every $p \in M$ can be uniquely written as $p=n a \cdot o\left(n \in N^{-}, a \in A\right)$. We regard the nilpotent group $N^{-}$as a boundary for the symmetric space $M$ in the following sense. The bounded harmonic functions $u$ on $M$ have boundary values on $N^{-}$, i.e. $\lim _{\log a \rightarrow \infty} u(n a \cdot o) \equiv \varphi(n)$ exists a.e. (relative to the Haar measure on $N^{-}$) and $\varphi \in L^{\infty}\left(N^{-}\right) \cdot \log a \rightarrow \infty$ is understood with respect to the ordering induced on a by $a^{+}$. Moreover,

$$
u(n a \cdot o)=\varphi * P_{a}(n)=\int_{N^{-}} \varphi\left(n_{1}\right) P_{a}\left(n_{1}^{-1} n\right) d n_{1} .
$$

Received by the editors April 28, 1980.

AMS (MOS) subject classifications (1970). Primary 43A85, 53C35; Secondary 43A20, 43 A90. 
The function $P_{a}(n)$ on $N^{-} \times A$ is called the Poisson kernel for the symmetric space $M$ and is given by (Helgason [4])

$$
P_{a}(n)=c \varepsilon^{d / 2}\left[\left(\varepsilon+\frac{1}{2} Q\left(X_{-\alpha}\right)\right)^{2}+2 Q\left(X_{-2 \alpha}\right)\right]^{-d / 2}
$$

where

$$
\begin{aligned}
& n=\exp \left(X_{-\alpha}+X_{-2 \alpha}\right), \quad X_{-\alpha} \in \mathfrak{g}_{-\alpha}, \quad X_{-2 \alpha} \in \mathfrak{g}_{-2 \alpha} ; \\
& \varepsilon=e^{-\alpha(\log a)} ; \quad Q(X)=(X, X)_{\theta} / 2\left(m_{\alpha}+4 m_{2 \alpha}\right)
\end{aligned}
$$

with $(X, X)_{\theta}=-B(X, \theta X)$ for $X \in \mathfrak{g}, \theta$ denoting the Cartan involution associated with the pair $(\mathfrak{g}, \mathfrak{f}) ; m_{\alpha}=\operatorname{dim} g_{-\alpha}, m_{2 \alpha}=\operatorname{dim} g_{-2 \alpha}, d=m_{\alpha}+2 m_{2 \alpha}$. The constant $c$ is such that the integral of $P_{a}$ over $N^{-}$is equal to 1 .

The following theorem on "tangential" convergence for bounded harmonic functions on the Siegel domain

$$
D_{r-1}=\left\{\left(z_{1}, \ldots, z_{r}\right) \in \mathbf{C}^{r}: \operatorname{Im} z_{r}>\sum_{j=1}^{r-1}\left|z_{j}\right|^{2}\right\},
$$

$r \geqslant 2$, (or, equivalently, on $M=S U(r, 1) / S(U(r) \times U(1)$ )-the complex hyperbolic space) has been obtained by Hulanicki and Ricci [5]. We formulate it below in terms of a homogeneous space $M$.

THEOREM. Let $u$ be a bounded harmonic function on a noncompact rank one symmetric space $M$. In the notation above, assume that for an $a_{0} \in A$, $\lim _{N^{-} \ni n \rightarrow \infty} u\left(n a_{0} \cdot o\right)=c_{0}$. Then for any $a \in A, \lim _{N^{-} \ni n \rightarrow \infty} u(n a \cdot o)=c_{0}$.

Our aim here is to prove the above Theorem and the proof is based on the classification of symmetric spaces. That is, we discuss separately the cases of $M$ being the real, complex (to see how the $M=D_{r-1}$ case fits to our scheme), quaternion and octonion hyperbolic space, which corresponds respectively to $G$ being the classical group $S O_{0}(r, 1), S U(r, 1), S p(r, 1)$ and the exceptional group $F_{4(-20)}$. Following the Hulanicki-Ricci method, for each case we construct a suitable commutative subalgebra $Q$ of (multi) radial functions in $L^{1}\left(N^{-}\right)$, to which the Poisson kernel $P_{a}$ belongs. We describe the set $\mathfrak{M}(\mathscr{Q})$ of the maximal ideals in $Q$ and check that the Gel'fand transform $\hat{P}_{a}$ of $P_{a}$ never vanishes on $\mathfrak{M}(\mathbb{Q})$. The Theorem may then be stated as a theorem on certain ideals in $L^{1}\left(N^{-}\right)$and is a consequence of the Wiener property of the algebra $\mathbb{Q}$. To study the algebra $\mathbb{Q}$ we use the holomorphically induced (realizations of the irreducible unitary) representations of $N^{-}$.

1. Nilpotent group $\mathrm{N}^{-}$. Let $\mathbf{F}$ denote the field $\mathbf{R}, \mathbf{C}, \mathbf{H}$ or the Cayley numbers $\mathbf{O}$ (octonions); $\mathbf{F}_{0}=\{q \in \mathbf{F}: q+\bar{q}=0\}$, ${ }^{-}$being the usual conjugation in $\mathbf{F}=\mathbf{C}, \mathbf{H}$, $\mathbf{O}$ and $\bar{q}=q$ for $q \in \mathbf{R} ; \operatorname{Im} q=\frac{1}{2}(q-\bar{q}), \sigma=2 s=\operatorname{dim}_{\mathbf{R}} \mathbf{F}$. According to the notation of the previous section, $g=\mathfrak{f}+\mathfrak{p}$ and for the classical $G$ we have (cf., e.g., $[3$, pp. 348-351]) 


$$
\begin{aligned}
& \mathfrak{l}=\left\{\left(\begin{array}{ll}
Z & 0 \\
0 & p
\end{array}\right): \begin{array}{c}
Z \text { an } r \times r \text { skew-Hermitian matrix over } \mathbf{F}, \\
p \in \mathbf{F}_{0}, \operatorname{tr} Z=-p \text { in case of } \mathbf{F}=\mathbf{C}
\end{array}\right\}, \\
& \mathfrak{p}=\left\{\left(\begin{array}{ll}
0 & { }^{t} q \\
\bar{q} & 0
\end{array}\right): q \in \mathbf{F}^{r}=\mathbf{F} \times \cdots \times \mathbf{F}\right\}, \\
& \mathfrak{a}=\left\{t E_{1, r+1}+t E_{r+1,1}: t \in \mathbf{R}\right\},
\end{aligned}
$$

where $E_{k l}$ denotes the $(r+1) \times(r+1)$ matrix $\left(\delta_{a k} \delta_{b l}\right)_{1<a, b<r+1}, r \geqslant 2$. We choose a basis $H=E_{1, r+1}+E_{r+1,1}$ in $a$ and fix an ordering so that $H \in a^{+}$. Then $\alpha \in a^{*}$ such that $\alpha(H)=1$ is a positive restricted root, and we have

$$
\begin{aligned}
& \mathfrak{g}_{-\alpha}=\left\{\left[\begin{array}{ccc}
0 & -\bar{q} & 0 \\
t & 0 & t \\
0 & \bar{q} & 0
\end{array}\right]: q=\left(q_{2}, \ldots, q_{r}\right) \in \mathbf{F}^{r-1}\right\}, \\
& g_{-2 \alpha}=\left\{\left[\begin{array}{ccc}
p & 0 & p \\
0 & 0 & 0 \\
\bar{p} & 0 & \bar{p}
\end{array}\right]: p \in \mathbf{F}_{0}\right\} \quad(=\{0\} \text { for } \mathbf{F}=\mathbf{R}) .
\end{aligned}
$$

We shall identify $\mathfrak{n}^{-}=g_{-\alpha} \oplus g_{-2 \alpha}$ with $\mathbf{F}^{r-1} \times \mathbf{F}_{0}$ by the correspondence

$$
\left[\begin{array}{ccc}
p & -\bar{q} & p \\
{ }^{t} q & 0 & { }^{t} q \\
\bar{p} & \bar{q} & \bar{p}
\end{array}\right] \leftrightarrow(q,-p) .
$$

In these coordinates on $\mathrm{n}^{-}$the commutator of $(q, p)=\left(q_{1}, \ldots, q_{r-1}, p\right)$ and $\left(q^{\prime}, p^{\prime}\right)=\left(q_{1}^{\prime}, \ldots, q_{r-1}^{\prime}, p^{\prime}\right)$ in $\mathbf{F}^{r-1} \times \mathbf{F}_{0}$ is given by

$$
\left[(q, p),\left(q^{\prime}, p^{\prime}\right)\right]=\left(0,2 \operatorname{Im}\left(\bar{q} \cdot q^{\prime}\right)\right),
$$

where we have put $\bar{q} \cdot q^{\prime}$ for $\sum_{l=1}^{r-1} \bar{q}_{l} q_{l}^{\prime}$. We also have the formula (cf., e.g., [11, p. 39])

$$
\left((q, p),\left(q^{\prime}, p^{\prime}\right)\right)_{\theta}=4\left(m_{\alpha}+4 m_{2 \alpha}\right) \operatorname{Re}\left(\bar{q} \cdot q^{\prime}+\bar{p} p^{\prime}\right) .
$$

For the exceptional $G$ (cf., e.g., [10, pp. 522-530]), $\mathfrak{g}=\mathfrak{f}_{4(-20)}$ is isomorphic to the Lie algebra $\operatorname{Der}(q)$ of derivations of the Jordan algebra $(q, \circ)$ of $3 \times 3$ octonion matrices $A$ of the form

$$
A=\left(\begin{array}{ccc}
\alpha_{1} & a_{3} & a_{2} \\
\bar{a}_{3} & \alpha_{2} & a_{1} \\
-\bar{a}_{2} & -\bar{a}_{1} & \alpha_{3}
\end{array}\right), \quad a_{i} \in \mathbf{O}, \quad \alpha_{i} \in \mathbf{R}, \quad i=1,2,3,
$$

with multiplication given by $A \circ B=\frac{1}{2}(A B+B A), A, B \in \mathcal{G}, A B$ denoting the usual matrix multiplication. We have

$$
\begin{aligned}
& \mathfrak{f}=\left\{D \in \operatorname{Der}(\mathcal{g}): D\left(E_{33}\right)=0\right\}, \\
& \mathfrak{p}=\left\{D_{Q} \in \operatorname{Der}(\mathcal{g}): Q=\left(\begin{array}{ll}
0 & t \\
\bar{q} & 0
\end{array}\right), \quad q \in \mathbf{O}^{2}\right\},
\end{aligned}
$$

where $D_{Q}(B)=Q B-B Q, B \in \mathcal{G}$. 


$$
a=\left\{D_{Q} \in \operatorname{Der}(\mathcal{F}): Q=t E_{13}+t E_{31}, \quad t \in \mathbf{R}\right\} .
$$

We choose $H=D_{Q}$ with $Q=E_{13}+E_{31} \in a^{+}$and $\alpha \in a^{*}$ such that $\alpha(H)=1$. Then

$$
\begin{aligned}
& g_{-\alpha}=\left\{D_{Q(q)}: Q(q)=\left[\begin{array}{ccc}
0 & -\bar{q} & 0 \\
q & 0 & q \\
0 & \bar{q} & 0
\end{array}\right], \quad q \in \mathbf{O}\right\}, \\
& g_{-2 \alpha}=\left\{D_{Q(p)}: Q(p)=\left[\begin{array}{ccc}
p & 0 & p \\
0 & 0 & 0 \\
\bar{p} & 0 & \bar{p}
\end{array}\right], \quad p \in \mathbf{O}_{0}\right\} .
\end{aligned}
$$

Identifying $D_{Q(q)}+D_{Q(p)}$ in $\mathfrak{g}_{-\alpha} \oplus \mathfrak{g}_{-2 \alpha}$ with $(q,-p)$ in $\mathbf{O} \times \mathbf{O}_{0}$, we obtain the same formulas for the commutator and the inner product of $(q, p)$ and $\left(q^{\prime}, p^{\prime}\right)$ in $\mathbf{O} \times \mathbf{O}_{0}$ as those given by (1) and (2) above.

Writing $N^{-}$as the manifold $\mathfrak{n}^{-}$with the group multiplication given by the Campbell-Hausdorff formula we obtain

Proposition 1. The underlying manifold for the nilpotent group $N^{-}$is $\mathbf{F}^{r-1} \times \mathbf{F}_{0}$ with $r \geqslant 2$ for $\mathbf{F}=\mathbf{R}, \mathbf{C}, \mathbf{H}\left(\mathbf{F}_{0}=\{0\}\right.$ if $\left.\mathbf{F}=\mathbf{R}\right)$ and with $r=2$ for $\mathbf{F}=\mathbf{O}$. The group law is

$$
(q, p)\left(q^{\prime}, p^{\prime}\right)=\left(q+q^{\prime}, p+p^{\prime}+\operatorname{Im}\left(\bar{q} \cdot q^{\prime}\right)\right) .
$$

The Haar measure on $N^{-}$is the ordinary Lebesgue measure on $\mathbf{R}^{k} \approx \mathbf{F}^{r-1} \times \mathbf{F}_{0}$, $k=r \sigma-1$. We normalize it so that the volume of the unit cube in $\mathbf{R}^{k}$ is 1 and denote by dqdp. The Poisson kernel is given by

$$
P_{\exp (t H)}(q, p)=c_{r, \mathbf{F}} \varepsilon^{d / 2}\left[\left(|q|^{2}+\varepsilon\right)^{2}+4|p|^{2}\right]^{-d / 2},
$$

where $\varepsilon=e^{-t}, d=(r+1) \sigma-2,|q|^{2}=\bar{q} \cdot q, c_{r, \mathbf{F}}=2^{d-1} \pi^{-r s} \Gamma(r s)$ with $\sigma=2 s=$ $\operatorname{dim}_{\mathbf{R}} \mathbf{F}$.

2. Holomorphically induced representations of $N^{-}$. The adjoint and coadjoint action of $N^{-}$on $\mathfrak{n}^{-}$and $\mathfrak{n}^{-*}$, respectively, is given by

$$
\begin{aligned}
\operatorname{Ad}_{(q, p)}\left(q^{\prime \prime}, p^{\prime \prime}\right) & =\left(q^{\prime \prime}, p^{\prime \prime}+2 \operatorname{Im}\left(\bar{q} \cdot q^{\prime \prime}\right)\right), \\
\operatorname{Ad}_{(q, p)}^{*}\left(q^{\prime}, p^{\prime}\right) & =\left(q^{\prime}+2 q \bar{p}^{\prime}, p^{\prime}\right),
\end{aligned}
$$

$(q, p) \in N^{-},\left(q^{\prime \prime}, p^{\prime \prime}\right) \in \mathrm{n}^{-},\left(q^{\prime}, p^{\prime}\right) \in \mathrm{n}^{-*}, q \bar{p}^{\prime}=\left(q_{1} \bar{p}^{\prime}, \ldots, q_{r-1} \bar{p}^{\prime}\right)$, and we have identified $n^{-*}$, the dual space of $n^{-}$, with $\mathfrak{n}^{-}$by $\langle\cdot, \cdot\rangle=(\cdot, \cdot)_{\theta} / 4\left(m_{\alpha}+4 m_{2 \alpha}\right)$. The single points $\left(q^{\prime}, 0\right) \in \mathfrak{n}^{-*}$ are 0 -dimensional orbits of $\mathrm{Ad}^{*}$ on $\mathfrak{n}^{-*}$ and the corresponding (1-dimensional) representations of $N^{-}$are given by the characters

$$
\chi_{\left(q^{\prime}, 0\right)}(q, p)=\exp \left(\sqrt{-1} \operatorname{Re}\left(\bar{q}^{\prime} \cdot q\right)\right), \quad(q, p) \in N^{-} .
$$

The remaining (maximal dimensional) orbits, for $\mathbf{F}=\mathbf{C}, \mathbf{H}$ and $\mathbf{O}$, are of the form $\mathbf{F}^{-1} \times\left\{p^{\prime \prime}\right\}, p^{\prime \prime} \neq 0$, so they are parameterized, e.g., by the functionals $f=\left(0, p^{\prime \prime}\right)$ $\in \mathfrak{n}^{-*}$ with $p^{\prime \prime} \in F_{0} \backslash\{0\}$. For such $f$ and $(q, p),\left(q^{\prime}, p^{\prime}\right) \in \mathfrak{n}^{-}$we have

$$
\left\langle f,\left[(q, p),\left(q^{\prime}, p^{\prime}\right)\right]\right\rangle=2 \operatorname{Re}\left(\left(q p^{\prime \prime}\right)^{-} \cdot q^{\prime}\right)=-2 \operatorname{Re}\left(\bar{q} \cdot\left(q^{\prime} p^{\prime \prime}\right)\right),
$$


i.e. the operator $R_{p^{\prime \prime}}: q \rightarrow q p^{\prime \prime}$ is skew-symmetric on $\mathbf{F}^{r-1}$ with respect to the R-bilinear symmetric form $\langle\cdot, \cdot\rangle$ on $\mathbf{F}^{r-1}$ given by $\left\langle q, q^{\prime}\right\rangle=2 \operatorname{Re}\left(\bar{q} \cdot q^{\prime}\right)=$ the usual inner product on $\mathbf{R}^{k}, k=(r-1) \sigma$. Let $1, i$ denote the usual basis of $\mathbf{C}$ (over $\mathbf{R}) ; 1, i, j, k$ the basis of $\mathbf{H}$, and $1, i, j, k, e, i e, j e, k e$ the basis of $\mathbf{O}(=\mathbf{H}+\mathbf{H e}$ with the multiplication defined by $(a e) b=(a \bar{b}) e, a(b e)=(b a) e,(a e)(b e)=-\bar{b} a$ for $a, b \in \mathbf{H})$. Suppose now that $f=(0, i \lambda) \in \mathfrak{n}^{-*}$ with $\lambda$ positive real. In the above bases of $\mathbf{F}$ the matrix of $R_{i \lambda}$ acting on $\mathbf{F}^{r-1}$ with $r=2$ is equal to $\lambda\left(E_{21}-E_{12}\right)$ for $\mathbf{F}=\mathbf{C}$, to $\lambda\left(E_{21}-E_{12}\right)-\lambda\left(E_{43}-E_{34}\right)$ for $\mathbf{F}=\mathbf{H}$ and to $\lambda\left(E_{21}-E_{12}\right)-$ $\lambda\left(E_{43}-E_{34}\right)-\lambda\left(E_{65}-E_{56}\right)+\lambda\left(E_{87}-E_{78}\right)$ for $\mathbf{F}=\mathbf{O}$. Put

$$
\begin{array}{ll}
e_{1}=\frac{1}{2}(1+\sqrt{-1} i), & e_{2}=\frac{1}{2}(\sqrt{-1} j+k), \\
e_{3}=\frac{1}{2}(\sqrt{-1} e+i e), & e_{4}=\frac{1}{2}(j e+\sqrt{-1} k e)
\end{array}
$$

for elements in $\mathbf{F}^{\mathbf{C}}=\mathbf{F}+\sqrt{-1} \mathbf{F}$-the complexification of $\mathbf{F}$. Now define a subspace $W$ of $\mathbf{F}^{\mathbf{C}}$ by

$$
W=\left\{\begin{array}{l}
\mathbf{C} e_{1} \text { if } \mathbf{F}=\mathbf{C}, \\
\mathbf{C} e_{1}+\mathbf{C} e_{2} \text { if } \mathbf{F}=\mathbf{H}, \\
\mathbf{C} e_{1}+\mathbf{C} e_{2}+\mathbf{C} e_{3}+\mathbf{C} e_{4} \text { if } \mathbf{F}=\mathbf{O} .
\end{array}\right.
$$

Thus

$$
\mathfrak{h}=W^{r-1} \times \mathbf{F}_{0}^{\mathbf{C}}
$$

is a positive polarization at $f=(0, i \lambda)$ such that

$$
\mathfrak{h}+\overline{\mathfrak{h}}=\mathfrak{n}^{-\mathbf{c}}, \quad \mathfrak{h} \cap \overline{\mathfrak{h}}=\{0\} \times \mathbf{F}_{0}^{\mathbf{C}}, \quad \mathfrak{h} /\{0\} \times \mathbf{F}_{0}^{\mathbf{C}}=W,
$$

where $\bar{z}=x-\sqrt{-1} y$ for $z=x+\sqrt{-1} y \in \mathfrak{n}^{-}+\sqrt{-1} \mathfrak{n}^{-}=\mathfrak{n}^{-\mathbf{C}}$. For arbitrary $f=\left(0, p^{\prime \prime}\right)$ with $p^{\prime \prime} \in \mathbf{F}_{0} \times\{0\}$, there exists an orthogonal transformation $\Omega$ on $\mathbf{R}^{\boldsymbol{o}} \approx \mathbf{F}$, such that

$$
\left\langle R_{p^{\prime \prime}} q, q^{\prime}\right\rangle=\left\langle R_{i\left|p^{\prime \prime}\right|}{ }^{\prime} \Omega q,{ }^{\prime} \Omega q^{\prime}\right\rangle, \quad q, q^{\prime} \in \mathbf{F}^{r-1} .
$$

Hence,

$$
\left\langle f,\left[(q, p),\left(q^{\prime}, p^{\prime}\right)\right]\right\rangle=\left\langle\left(0, i\left|p^{\prime \prime}\right|\right),\left[\left({ }^{\prime} \Omega q, p\right),\left({ }^{\imath} \Omega q^{\prime}, p^{\prime}\right)\right]\right\rangle,
$$

and $\mathfrak{h}^{\prime}=\Omega \mathfrak{h}$, with $\mathfrak{h}$ as in (2.1), is a positive polarization at $f$ and $\mathfrak{h}^{\prime}$ satisfies (2.2) with $W^{\prime}=\Omega W$ instead of $W$. Here $\Omega\left(q+\sqrt{-1} q^{\prime}\right)$ is understood as $\Omega q$ $+\sqrt{-1} \Omega q^{\prime}, q, q^{\prime} \in \mathbf{F}^{r-1}$, and $\Omega q=\left(\Omega q_{1}, \ldots, \Omega q_{r-1}\right) \in \mathbf{F}^{r-1}$. As in [1, pp. 158162] one obtains that the space $\mathcal{H}(f, \mathfrak{h})$ of the representation $\rho(f, \mathfrak{h})$ corresponding to the chosen $f$ and $\mathfrak{h}^{\prime}$ may be realized as a space of complex $C^{\infty}$ functions $\psi$ on the complex space $\bar{W}^{\prime}$, square integrable with respect to the measure $\exp (-\sqrt{-1}\langle f,[Y, \bar{Y}]\rangle) d Y d \bar{Y}\left(d Y d \bar{Y}\right.$ denoting the Lebesgue measure) on $\bar{W}^{\prime}$ and satisfying the following functional equation:

$$
[\tau(\sqrt{-1} \bar{X}) \psi](\bar{Y})=\sqrt{-1}[\tau(\bar{X}) \psi](\bar{Y}), \quad X, Y \in W^{\prime},
$$


where $[\tau(\bar{X}) \psi](\bar{Y})=\left.(d / d t) \psi(\bar{Y}+t \bar{X})\right|_{t=0}$. The representation $\rho$ is given by

$$
\begin{aligned}
& {\left[\rho(f, \mathfrak{h})\left(\exp \left(\bar{X}_{0}+X_{0}+Z_{0}\right)\right) \psi\right](\bar{X})} \\
& \quad=\exp \left(\sqrt{-1}\left\langle f,\left[X_{0}, \bar{X}\right]\right\rangle-(\sqrt{-1} / 2)\left\langle f,\left[X_{0}, \bar{X}_{0}\right]\right\rangle\right) x_{f}\left(Z_{0}\right) \cdot \psi\left(\bar{X}-\bar{X}_{0}\right),
\end{aligned}
$$

where $\bar{X}_{0}+X_{0}+Z_{0}$ is in $\mathfrak{n}^{-}$for $X_{0} \in W^{\prime}, Z_{0} \in \mathbf{F}_{0} ; \chi_{f}\left(Z_{0}\right)=\exp \left(\sqrt{-1}\left\langle f, Z_{0}\right\rangle\right)$. Passing to the complex coordinates $\left(z_{1}, \ldots, z_{s(r-1)}\right)$ on $\bar{W}^{\prime}$, according to the identifications

$$
\begin{aligned}
& \bar{X}=\left(z_{1} \bar{e}_{1}, \ldots, z_{r-1} \bar{e}_{1}\right) \leftrightarrow\left(z_{1}, \ldots, z_{r-1}\right) \quad \text { for } \mathbf{F}=\mathbf{C}, \\
& \bar{X}=\left(z_{1} \bar{e}_{1}+z_{2} \bar{e}_{2}, \ldots, z_{2(r-1)-1} \bar{e}_{1}+z_{2(r-1)} \bar{e}_{2}\right) \leftrightarrow\left(z_{1}, \ldots, z_{2(r-1)}\right) \quad \text { for } \mathbf{F}=\mathbf{H}, \\
& \bar{X}=\left(z_{1} \bar{e}_{1}+z_{2} \bar{e}_{2}+z_{3} \bar{e}_{3}+z_{4} \bar{e}_{4}\right) \leftrightarrow\left(z_{1}, \ldots, z_{4}\right) \quad \text { for } \mathbf{F}=\mathbf{O},
\end{aligned}
$$

we have

$$
\begin{gathered}
\sqrt{-1}\left\langle f,\left[X, \bar{X}^{\prime}\right]\right\rangle=\left|p^{\prime \prime}\right| \bar{z} \cdot z^{\prime}, \quad X=(\bar{X})^{-}, \quad \bar{X}, \bar{X}^{\prime} \in \bar{W}^{\prime}, \\
f=\left(0, p^{\prime \prime}\right), \quad z=\left(z_{1}, \ldots, z_{s(r-1)}\right) \in \mathbf{C}^{s(r-1)} .
\end{gathered}
$$

Rewriting (2.3) in these coordinates we obtain

Proposition 2.1. All the inequivalent irreducible unitary representations of $\mathrm{N}^{-}$fall into two classes:

(a) a family of 1-dimensional characters $\chi_{q^{\prime}}$ parameterized by $q^{\prime} \in \mathrm{F}^{r-1}$ and given by

$$
\chi_{q^{\prime}}(q, p)=\exp \left(\sqrt{-1} \operatorname{Re}\left(\bar{q}^{\prime} \cdot q\right)\right), \quad(q, p) \in N^{-}
$$

(b) a family of infinite-dimensional representations $\rho_{p^{\prime}}$ parameterized by $p^{\prime} \in$ $F_{0} \backslash\{0\}$. The Hilbert space $\mathcal{H}_{p^{\prime}}$ of the representation $\rho_{p^{\prime}}$ consists of holomorphic functions $\psi$ on $\mathbf{C}^{s(r-1)}$, such that

$$
\|\psi\|_{p^{\prime}}^{2}=\int_{\mathbf{C}^{(r-1)}}|\psi(z)|^{2} \exp \left(-\left|p^{\prime}\right||z|^{2}\right) d z d \bar{z}<\infty
$$

with

$$
d z d \bar{z}=\prod_{j=1}^{s(r-1)} 2 d \operatorname{Re} z_{j} d \operatorname{Im} z_{j} .
$$

The action of $\rho_{p^{\prime}}$ on $\psi \in \mathcal{H}_{p^{\prime}}$ is given by

$$
\left(\rho_{p^{\prime}}(q, p) \psi\right)\left(z_{j}=\exp \left(\sqrt{-1} \operatorname{Re}\left(\bar{p}^{\prime} p\right)+\left|p^{\prime}\right|\left(\bar{z}_{0} \cdot z-\frac{1}{2}\left|z_{0}\right|^{2}\right)\right) \psi\left(z-z_{0}\right),\right.
$$

$z \in \mathbf{C}^{(r-1)},(q, p) \in N^{-}$with $q=q\left(z_{0}\right), z_{0} \in \mathbf{C}^{(r-1)}$, where

$$
q(z)=\Omega\left(P\left(z_{1}, \ldots, z_{s}\right), \ldots, P\left(z_{s(r-2)+1}, \ldots, z_{s(r-1)}\right)\right)
$$

and $P\left(z_{s(l-1)+1}, \ldots, z_{s l}\right), l=1, \ldots, r-1$, is defined as

$$
\operatorname{Re} z_{l}+i \operatorname{Im} z_{l} \text { for } \mathbf{F}=\mathbf{C},
$$

$$
\begin{aligned}
\operatorname{Re} z_{2 l-1} & +i \operatorname{Im} z_{2 l-1}+j \operatorname{Im} z_{2 l}+k \operatorname{Re} z_{2 l} \quad \text { for } \mathbf{F}=\mathbf{H} \\
\operatorname{Re} z_{1} & +i \operatorname{Im} z_{1}+j \operatorname{Im} z_{2}+k \operatorname{Re} z_{2}+e \operatorname{Im} z_{3} \\
& +i e \operatorname{Re} z_{3}+j e \operatorname{Re} z_{4}+k e \operatorname{Im} z_{4} \quad \text { for } \mathbf{F}=\mathbf{O}
\end{aligned}
$$


$\operatorname{Im}(a+\sqrt{-1} b)=b, a, b \in \mathbf{R}$. The functions

$$
\psi_{n}^{p^{\prime}}(z)=(2 \pi)^{-m / 2}\left|p^{\prime}\right|^{(|n|+m) / 2}(n !)^{-1 / 2} z^{n}, \quad z \in \mathbf{C}^{m},
$$

$n=\left(n_{1}, \ldots, n_{m}\right) \in \mathbf{N}^{m}$, with $n !=n_{1} ! \cdots n_{m} !, z^{n}=z_{1}^{n_{1}} \cdots z_{m}^{n_{m}}, \quad|n|=n_{1}$

$+\cdots+n_{m}, m=s(r-1)$, form an orthonormal basis of $\mathcal{H}_{p^{\prime}}$ as $n$ runs over $\mathbf{N}^{m}$.

We also note the following symmetry properties of $\chi_{q^{\prime}}$ and $\rho_{p^{\prime}}$ relative to the orthogonal and the unitary transformations.

Proposition 2.2. (a) Let $o_{1}, \ldots, o_{r-1} \in O(\sigma, \mathbf{R})$; then for $q^{\prime} \in \mathbf{F}^{r-1}$,

$$
\chi_{q}\left({ }^{t} o_{1} q_{1}, \ldots,{ }^{t} o_{r-1} q_{r-1}, p\right)=\chi_{\left(o_{1} q_{1}^{\prime}, \ldots, o_{r-1} q_{r-1}^{\prime}\right)}(q, p), \quad(q, p) \in N^{-} .
$$

(b) Let $u_{1}, \ldots, u_{r-1} \in U(s)$; then for $p^{\prime} \in F_{0} \backslash\{0\}$,

$$
A_{u}^{-1} \rho_{p^{\prime}}(q, p) A_{u}=\rho_{p^{\prime}}\left(q^{u}, p\right) \text {, }
$$

$(q, p) \in N^{-}$with $q=q\left(z_{0}\right), \quad z_{0} \in \mathrm{C}^{s(r-1)}$ and $q^{u}=q\left(u z_{0}\right)$ with $u z=$ $\left(u_{1}\left(z_{1}, \ldots, z_{s}\right), \ldots, u_{r-1}\left(z_{s(r-2)+1}, \ldots, z_{s(r-1)}\right)\right) ;\left(\left(A_{u}\right) \psi\right)(z)=\psi(u z)$ for $\psi \in \mathcal{H}_{p^{\prime}}$; $u=\left(u_{1}, \ldots, u_{r-1}\right), \sigma=2 s=\operatorname{dim}_{\mathbf{R}} \mathbf{F}$.

\section{Algebra of multiradial functions.}

Definition (CF. Geller [2]). We say that a function $F$ on $N^{-}=F^{r-1} \times F_{0}$, $\mathbf{F}=\mathbf{C}, \mathbf{H}, \mathbf{O}$, is multiradial if there is a function $f$ on $\mathbf{R}_{+}^{r-1} \times \mathbf{F}_{0}$ such that

$$
F(q, p)=f\left(\left|q_{1}\right|, \ldots,\left|q_{r-1}\right|, p\right), \quad(q, p) \in N^{-} .
$$

Proposition 3.1. Let $Q$ denote the space of multiradial functions in $L^{1}\left(N^{-}\right)$. Then $\mathbb{Q}$ is a commutative closed ${ }^{*}$-subalgebra of $L^{1}\left(N^{-}\right)$and $\mathbb{Q}$ is symmetric.

Proof. $1^{\circ}$. If $F, G \in \mathbb{Q}$ then $F * G \in \mathbb{Q}$. For we have

$$
\begin{aligned}
F * G\left(q^{\prime}, p^{\prime}\right)= & \int f\left(\left|q_{1}\right|, \ldots,\left|q_{r-1}\right|, p\right) \\
& \times g\left(\left|q_{1}^{\prime}-q_{1}\right|, \ldots,\left|q_{r-1}^{\prime}-q_{r-1}\right|, p^{\prime}-p-\operatorname{Im}\left(\bar{q} \cdot q^{\prime}\right)\right) d q d p .
\end{aligned}
$$

Substituting $q=\left(\left(q_{1}^{\prime} /\left|q_{1}^{\prime}\right|\right) \tilde{q}_{1}, \ldots,\left(q_{r-1}^{\prime} /\left|q_{r-1}^{\prime}\right|\right) \tilde{q}_{r-1}\right)$ we get (since $(a \bar{b}) b=a(\bar{b} b)$ for $a, b \in \mathbf{F}$ )

$$
\begin{aligned}
\int f\left(\left|\tilde{q}_{1}\right|, \ldots,\left|\tilde{q}_{r-1}\right|, p\right) g\left(\left|q_{1}^{\prime}\right|\left|1-\tilde{q}_{1} /\right| q_{1}^{\prime}||, \ldots,\right. & \\
& \left.\left|q_{r-1}^{\prime}\right|\left|1-\tilde{q}_{r-1} /\right| q_{r-1}^{\prime}||, p^{\prime}-p-\operatorname{Im}\left(\sum_{l=1}^{r-1} \overline{\tilde{q}}_{l}\left|q_{l}^{\prime}\right|\right)\right) d \tilde{q} d p,
\end{aligned}
$$

i.e. $F * G$ is multiradial. Obviously $\mathbb{Q}$ is closed.

$2^{\circ} . Q$ is commutative (cf. Kaplan and Putz [6, p. 377]). Under the orthogonal change of variables

$$
q_{l} \mapsto q_{l}^{\prime \prime}=q_{l}^{\prime} \cdot 2 \operatorname{Re}\left(\bar{q}_{l} q_{l}^{\prime}\right) /\left|q_{l}^{\prime}\right|^{2}-q_{l}, \quad l=1, \ldots, r-1,
$$


one has $\left|q_{l}^{\prime}-q_{l}^{\prime \prime}\right|=\left|q_{l}^{\prime}-q_{l}\right|$ and $\operatorname{Im}\left(\bar{q}_{l} q_{l}^{\prime}\right)=-\operatorname{Im}\left(\bar{q}_{l}^{\prime \prime} q_{l}^{\prime}\right)$. Thus (3.1) is equal to

$$
\begin{aligned}
\int f\left(\left|q_{1}^{\prime \prime}\right|, \ldots,\right. & \left.\left|q_{r-1}^{\prime \prime}\right|, p\right) \\
& \times g\left(\left|q_{1}^{\prime}-q_{1}^{\prime \prime}\right|, \ldots,\left|q_{r-1}^{\prime}-q_{r-1}^{\prime \prime}\right|, p^{\prime}-p-\sum_{l=1}^{r-1}-\operatorname{Im}\left(\bar{q}_{l}^{\prime \prime} q_{l}^{\prime}\right)\right) d q^{\prime \prime} d p \\
& =\int_{N^{-}} F\left(q^{\prime \prime}, p\right) G\left(\left(q^{\prime}, p^{\prime}\right)\left(q^{\prime \prime}, p\right)^{-1}\right) d q^{\prime \prime} d p \\
& =G * F\left(q^{\prime}, p^{\prime}\right) .
\end{aligned}
$$

$3^{\circ}$. Since $L^{1}\left(N^{-}\right)$is symmetric (Leptin [8, p. 205]), its *-subalgebra $\mathcal{Q}$ is also symmetric.

Proposition 3.2. For $F \in \mathbb{Q}$ and $u=\left(u_{1}, \ldots, u_{r-1}\right) \in U(s) \times \cdots \times U(s)$, the operators $\rho_{p^{\prime}}(F)=\int_{N^{-}} F(q, p) \rho_{p^{\prime}}(q, p) d q d p$ and $A_{u}$ commute on $\mathcal{K}_{p^{\prime}}$.

Proof. By Proposition 2.2(b),

$$
\begin{aligned}
A_{u}^{-1} \int \rho_{p^{\prime}} & \left(q\left(z_{0}\right), p\right) F\left(q\left(z_{0}\right), p\right) d q\left(z_{0}\right) d p A_{u} \\
= & \int \rho_{p^{\prime}}\left(q\left(u z_{0}\right), p\right) F\left(q\left(z_{0}\right), p\right) d q\left(z_{0}\right) d p .
\end{aligned}
$$

Since

$$
q_{l}\left(u z_{0}\right)=\Omega\left(P\left(u_{l} Z_{l}\right)\right)=\left(\Omega P u_{l} P^{-1} \Omega^{-1}\right)\left(\Omega P Z_{l}\right)
$$

with $Z_{l}=\left(z_{s(l-1)+1}^{0}, \ldots, z_{s l}^{0}\right), l=1, \ldots, r-1$, and since $\Omega P u_{l} P^{-1} \Omega^{-1}$ is an orthogonal transformation on $\mathbf{R}^{\sigma} \approx \mathbf{F}$, and $\Omega P Z_{l}=q_{l}\left(z_{0}\right)$, the last integral is equal to $\rho_{p^{\prime}}(F)$.

REMARK. For $\mathbf{F}=\mathbf{R}$, the corresponding group $N^{-}$is $\mathbf{R}^{r-1}$, so the algebra $L^{1}\left(N^{-}\right)$is already commutative, and, as in the case $M=\mathbf{R}^{n} \times \mathbf{R}_{+}$with the Euclidean metric [5], we consider $Q=L^{1}\left(N^{-}\right)$.

4. Multiplicative linear functionals on $Q$. Let $\Phi$ be a nonzero multiplicative linear functional on $\mathcal{Q}$. Since $\mathcal{Q}$ is a symmetric *-subalgebra of $L^{1}\left(N^{-}\right)$, there exist an irreducible ${ }^{*}$-representation $\pi$ of $L^{1}\left(N^{-}\right)$and a unit vector $\xi$ in the Hilbert space $\mathcal{H}_{\pi}$ such that

$$
\pi(F) \xi=\Phi(F) \xi \quad \text { for all } F \text { in } \mathcal{Q} .
$$

If $\mathcal{H}_{\pi}$ is one dimensional, then

$$
\pi(F) \xi=\int_{N^{-}} F(q, p) \chi_{q^{\prime}}(q, p) d q d p \xi
$$

for some $q^{\prime} \in \mathbf{F}^{r-1}$, and by Proposition 2.2(a), if $q^{\prime}$ and $q^{\prime \prime}$ in $\mathbf{F}^{r-1}$ are such that $\left|q_{l}^{\prime}\right|=\left|q_{l}^{\prime \prime}\right|, l=1, \ldots, r-1$, the $\Phi$ 's corresponding by (4.1) and (4.2) to $\chi_{q^{\prime}}$ and $\chi_{q^{\prime \prime}}$ are identical. If $\pi \approx \rho_{p^{\prime}}$, then by Proposition 3.2, $\rho_{p^{\prime}}(F)$ and $A_{u}$ commute. Now for $\psi(z)=\psi_{1}\left(Z_{1}\right) \ldots \psi_{r-1}\left(Z_{r-1}\right)$ with $z=\left(Z_{1}, \ldots, Z_{r-1}\right), Z_{l}=\left(z_{s(l-1)+1}, \ldots, z_{s l}\right), l$ $=1, \ldots, r-1$, we have

$$
\left(A_{u} \psi\right)(z)=\psi_{1}\left(u_{1} Z_{1}\right) \ldots \psi_{r-1}\left(u_{r-1} Z_{r-1}\right)
$$


Thus putting $\psi_{l}\left(Z_{l}\right)=Z_{l}^{n_{l}}$ with $n_{l}=\left(n_{1 l}, \ldots, n_{s l}\right) \in \mathrm{N}^{s}$, we note that $A_{u}$ preserves the finite-dimensional subspaces of $\mathcal{H}_{p^{\prime}}$, namely the spaces $\mathcal{H}^{n}=\bigotimes_{l=1}^{r-1} \mathcal{H}^{\left|n_{l}\right|}$, where $n=\left(\left|n_{1}\right|, \ldots,\left|n_{r-1}\right|\right) \in \mathbf{N}^{r-1}$. $\mathcal{H}^{\left|n_{1}\right|}$ is the space of homogeneous polynomials in $z_{s(l-1)}, \ldots, z_{s l}$ of degree $\left|n_{l}\right|$. Moreover, $\mathcal{H}_{p^{\prime}}=\bigoplus_{n} \mathcal{H}^{n}$-an orthogonal direct sum over $n \in \mathbf{N}^{r-1}$. We also note that $A_{u}$ restricted to $\mathcal{H}^{n}$ is equal to $\bigotimes_{l=1}^{r-1} T^{\left|n_{l}\right|}\left(u_{1}^{-1}, \ldots, u_{r-1}^{-1}\right)$ with $T^{k}, k=\left|n_{l}\right|$, being the representation of $U(s)$ on $\mathcal{F}^{k}$ given by $\left(T_{u}^{k} \psi\right)(Z)=\psi\left(u^{-1} Z\right)$. Since $T^{k}$ is irreducible (cf., e.g., [13, pp. 204-209]), the representations $T^{n}=\bigotimes_{l} T^{\left|n_{l}\right|}$ of $U(s) \times \cdots \times U(s), r-1$ copies of $U(s)$, act irreducibly on $\mathcal{H}^{n}$, and $T^{n} \approx T^{m}$ iff $n=m$. Hence, by Schur's Lemma, every intertwining operator $S$ for $\bigoplus_{n} T^{n}$ on $\mathcal{H}_{p^{\prime}}$ is of the form $S=\bigoplus_{n} c_{n}(S) \operatorname{Id}_{\mathcal{F}^{n}}$. In particular, each $\rho_{p^{\prime}}(F)$ with $F \in Q$ is such. It follows from (4.1) that $\Phi(F)$ is equal to one of the constants $c_{n}\left(\rho_{p^{\prime}}(F)\right), n \in \mathrm{N}^{r-1}$. Conversely, for every fixed $n$, the mapping $F \mapsto c_{n}\left(\rho_{p^{\prime}}(F)\right)$ defines a multiplicative linear functional on $\mathbb{Q}$. Now we shall derive explicit formulas for the constants $c_{n}$ above. Since, e.g.,

$$
c_{n}\left(\rho_{p^{\prime}}(F)\right)=\left(\rho_{p^{\prime}}(F) \psi_{n^{\prime}}^{p^{\prime}}, \psi_{n^{\prime}}^{p^{\prime}}\right)_{\mathcal{T}_{p^{\prime}}}
$$

with $n^{\prime}=\left(n_{1}, 0, \ldots, 0 ; n_{2}, 0, \ldots, 0 ; \ldots ; n_{r-1}, 0, \ldots, 0\right) \in\left(\mathbf{N}^{s}\right)^{r-1}$, we calculate the integral, see Proposition 2.1(b),

$$
\int_{\mathbf{C}^{(r-1)}}\left[\rho_{p^{\prime}}(F) \psi_{n^{\prime}}^{p^{\prime}}\right](z) \bar{\psi}_{n^{\prime}}^{p^{\prime}}(z) \exp \left(-\left|p^{\prime}\right||z|^{2}\right) d z d \bar{z}
$$

which in expanded form is equal to (with $k=s(r-1)$ )

$$
\begin{aligned}
(2 \pi)^{-k}(n !)^{-1}\left|p^{\prime}\right|^{|n|+k} & \\
\quad \times \int_{\mathbf{C}^{k}} \int_{\mathbf{F}^{r-1} \times \mathbf{F}_{0}} F\left(q\left(z_{0}\right)\right. & , p) \exp \left(\sqrt{-1} \operatorname{Re}\left(\bar{p}^{\prime} p\right)+\left|p^{\prime}\right|\left(\bar{z}_{0} \cdot z-\frac{1}{2}\left|z_{0}\right|^{2}\right)\right) \\
& \times\left(z-z_{0}\right)^{n^{\prime}} \bar{z}^{n^{\prime}} \exp \left(-\left|p^{\prime}\right||z|^{2}\right) d q\left(z_{0}\right) d p d z d \bar{z}
\end{aligned}
$$

The integral

$$
\int_{\mathbf{C}^{s(r-1)}}\left(z-z_{0}\right)^{n^{\prime}} \bar{z}^{n^{\prime}} \exp \left(-\left|p^{\prime}\right||z|^{2}\right) \exp \left(\left|p^{\prime}\right| \bar{z}_{0} \cdot z\right) d z d \bar{z}
$$

is equal to

$$
\begin{gathered}
\left(2 \pi /\left|p^{\prime}\right|\right)^{(s-1)(r-1)} \prod_{l=1}^{r-1} 2 \pi n_{l} !\left|p^{\prime}\right|^{-n_{l}-1} \sum_{j=0}^{n_{l}}\left(-\left|p^{\prime}\right|\left|z_{s(l-1)+1}^{0}\right|^{2}\right)^{j}\left(\begin{array}{c}
n_{l} \\
j
\end{array}\right)(j !)^{-1} \\
=(2 \pi)^{s(r-1)}\left|p^{\prime}\right|^{-|n|-s(r-1)} n ! \prod_{l=1}^{r-1} L_{n_{l}}\left(\left|p^{\prime}\right|\left|z_{s(l-1)+1}^{0}\right|^{2}\right)
\end{gathered}
$$

with $L_{n}$ being the Laguerre polynomial. (4.5a) is obtained (see [5]) by substituting the binomial formula for $\left(z-z_{0}\right)^{n}$, developing $\exp \left(-\left|p^{\prime}\right| \bar{z}_{0} \cdot z\right)$ in a power series and integrating this series term by term using the orthogonality relations for the 
functions $z^{n}$ in $\mathcal{H}_{p^{\prime}}$. Substituting (4.5a) in (4.4) we obtain that (4.3) is equal to

$$
\begin{aligned}
\int_{\mathbf{F}^{r-1} \times \mathbf{F}_{0}} F\left(q\left(z_{0}\right), p\right) & \exp \left(\sqrt{-1} \operatorname{Re}\left(\bar{p}^{\prime} p\right)-\frac{1}{2}\left|p^{\prime}\right|\left|z_{0}\right|^{2}\right) \\
& \times \prod_{l=1}^{r-1} L_{n_{l}}\left(\left|p^{\prime}\right|\left|z_{s(l-1)+1}^{0}\right|^{2}\right) d q\left(z_{0}\right) d p \\
= & \int_{0}^{\infty} d t_{1} \ldots \int_{0}^{\infty} d t_{r-1}\left(\int_{\mathbf{F}_{0}} f\left(t_{1}, \ldots, t_{r-1}, p\right) \exp \left(\sqrt{-1} \operatorname{Re}\left(\bar{p}^{\prime} p\right)\right) d p\right) \\
& \times \exp \left(-\frac{1}{2}\left|p^{\prime}\right|\left(t_{1}^{2}+\ldots+t_{r-1}^{2}\right)\right) \prod_{l=1}^{r-1} t_{l}^{\sigma-1} g_{l},
\end{aligned}
$$

with $f$ as in (3.0) and $\mathscr{G}_{l}$ given by

$$
\begin{aligned}
& g_{l}=\int_{S(\sigma-1)} L_{n_{l}}\left(\left|p^{\prime}\right|\left|z_{1}\right|^{2}\right) d S(q(Z)), \\
& Z=\left(z_{1}, \ldots, z_{s}\right) \in \mathbf{C}^{s}, \quad|Z|=t_{l},
\end{aligned}
$$

$S(\sigma-1)$ being the unit sphere in F. Since here $q(Z)=\Omega(P Z) / t_{l}$, with $P$ as in Proposition 2.1(b) and $\Omega \in O(\sigma, \mathbf{R})$, in order to compute $G_{l}$ one has to calculate the integrals

$$
\int_{S(\sigma-1)}\left|z_{1}(q)\right|^{2 j} d S(q), \quad j=0, \ldots, n_{l},
$$

where $\left|z_{1}(q)\right|^{2}=t_{l}^{2}\left(\left(q^{1}\right)^{2}+\left(q^{i}\right)^{2}\right)$ with $q^{1}, q^{i}, \ldots$ denoting the coordinates of $q$ in the (standard) basis $\{1, i, \ldots\}$ of $\mathbf{F}$ over $\mathbf{R}$. Now (4.6) is equal to

$$
\begin{gathered}
\int_{0}^{\pi / 2} \cos ^{2 j} \theta \cos \theta \sin ^{\sigma-3} \theta d \theta \cdot 2 \pi \cdot 2 \pi^{s-1}[(s-2) !]^{-1} t_{l}^{2 j} \\
=2 \pi^{s} t_{l}^{2 j} j ! /(j+s-1) ! .
\end{gathered}
$$

We summarize the results of this section in the following:

Proposition 4. The multiplicative linear functionals on $\mathbb{Q}$ fall into two classes:

(a) the functionals corresponding to $(r-1)$-tuples $\left(t_{1}, \ldots, t_{r-1}\right)$ of nonnegative real numbers and given by

$$
F \mapsto \hat{F}\left(t_{1}, \ldots, t_{r-1}\right)=\int_{\mathbf{F}^{r-1} \times \mathbf{F}_{0}} F(q, p) \exp \left(\sqrt{-1} \operatorname{Re}\left(\bar{q}^{\prime} \cdot q\right)\right) d q d p
$$

with $q^{\prime} \in \mathbf{F}^{r-1}$ arbitrary provided $\left(\left|q_{1}^{\prime}\right|, \ldots,\left|q_{r-1}^{\prime}\right|\right)=\left(t_{1}, \ldots, t_{r-1}\right)$.

(b) the functionals corresponding to pairs $\left(p^{\prime}, n\right) \in \mathbf{F}_{0} \backslash\{0\} \times \mathbf{N}^{r-1}$ and given by

$$
\begin{aligned}
F \mapsto \hat{F}\left(p^{\prime}, n\right)= & \left(2 \pi^{s}\right)^{r-1} \int_{\mathbf{R}_{+}^{r-1}} \exp \left(-\frac{\left|p^{\prime}\right|}{2}\left(t_{1}^{2}+\cdots+t_{r-1}^{2}\right)\right) \prod_{l=1}^{r-1} L_{n_{l}}^{(s-1)}\left(\left|p^{\prime}\right| t_{l}^{2}\right) t_{l}^{\sigma-1} \\
& \times\left(\int_{\mathbf{F}_{0}} f\left(t_{1}, \ldots, t_{r-1}, p\right) \exp \left(\sqrt{-1} \operatorname{Re}\left(\bar{p}^{\prime} p\right)\right) d p\right) d t_{1} \ldots d t_{r-1},
\end{aligned}
$$


where $f\left(\left|q_{1}\right|, \ldots,\left|q_{r-1}\right|, p\right)=F\left(q_{1}, \ldots, q_{r-1}, p\right)$ and

$$
\begin{aligned}
L_{k}^{(m)}(x) & =\sum_{j=0}^{k} \frac{(-x)^{j}}{(j+m) !}\left(\begin{array}{l}
k \\
j
\end{array}\right) \\
& =[(k+m) !]^{-1} x^{-m} e^{x}\left(d^{k} / d x^{k}\right)\left(x^{k+m} e^{-x}\right) .
\end{aligned}
$$

\section{Nonvanishing of the Gel'fand transform of $P_{a}$.}

LeMMA 1. For $1 \leqslant m<2 k+\frac{1}{2}, k>\frac{3}{2}$ and $Q>0$, the following formula holds:

$$
\begin{aligned}
& \int_{\mathbf{R}^{m}} \frac{\exp \left(\sqrt{-1} x_{0} \cdot x\right) d x}{\left(Q^{2}+4|x|^{2}\right)^{k}}= 2^{-m} \pi^{m / 2} \frac{\Gamma(k-m / 2)}{\Gamma(k)} Q^{m-2 k}, \text { for } x_{0}=0, \\
&= 2^{m+1-4 k} \pi^{(m+1) / 2} \frac{r^{2 k-m} e^{-(r / 2) Q}}{\Gamma(k) \Gamma(k-(m-1) / 2)} \\
& \times \int_{0}^{\infty} e^{-(r / 2) Q t}\left((t+1)^{2}-1\right)^{k-(m+1) / 2} d t, \\
& \text { for } r=\left|x_{0}\right| \neq 0 .
\end{aligned}
$$

Proof. For $x_{0}=0$, the integral is equal to the "area" of the unit sphere in $\mathbf{R}^{m}$ (=2 when $m=1$ ) times $\int_{\mathbf{R}_{+}} r^{m-1}\left(Q^{2}+4 r^{2}\right)^{-k} d r$ and we substitute $r=r^{\prime} Q / 2$. For $x_{0} \neq 0$, the function $\left[4\left(\left(\frac{1}{2} Q\right)^{2}+x^{2}\right)\right]^{-k}$ is radial on $\mathbf{R}^{m}$, hence its Fourier transform (5.1) is equal to, see, e.g., [9, p. 155],

$$
\begin{aligned}
& 4^{-k}(2 \pi)^{m / 2} r^{-(m-2) / 2} \int_{0}^{\infty}\left(\left(\frac{1}{2} Q\right)^{2}+t^{2}\right)^{-k} J_{(m-2) / 2}(r t) t^{m / 2} d t \\
& \quad m \geqslant 1, \quad k>3 / 2 .
\end{aligned}
$$

Combining now the Sonine formula [12, p. 434, (2)],

$$
\int_{0}^{\infty} \frac{x^{\nu+1} J_{\nu}(a x) d x}{\left(x^{2}+k^{2}\right)^{\mu+1}}=\frac{a^{\mu} k^{\nu-\mu}}{2^{\mu} \Gamma(\mu+1)} K_{\mu-\nu}(a k),
$$

valid when $-1<\operatorname{Re}(\nu)<2 \operatorname{Re}(\mu)+\frac{3}{2}$, with the following expression for the function $K[12$, p. 172, (4)],

$$
K_{\nu}(z)=\frac{\Gamma\left(\frac{1}{2}\right)\left(\frac{1}{2} z\right)^{\nu}}{\Gamma\left(\nu+\frac{1}{2}\right)} \int_{1}^{\infty} e^{-z t}\left(t^{2}-1\right)^{\nu-1 / 2} d t,
$$

valid for $\operatorname{Re}\left(\nu+\frac{1}{2}\right)>0,|\arg z|<\pi / 2$, we obtain (5.1).

LEMMA 2. For $\varepsilon>0, m>0, x \in \mathbf{R}^{n}$,

$$
\begin{aligned}
\left(\varepsilon+|x|^{2}\right)^{-m}=(4 \pi)^{-n / 2} \Gamma(m)^{-1} \int_{\mathbf{R}^{n}} \exp (-\sqrt{-1} x \cdot y) & \\
& \times\left(\int_{0}^{\infty} t^{m-1-m / 2} e^{-e t} e^{-|y|^{2} / 4 t} d t\right) d y,
\end{aligned}
$$

i.e. $\left(\varepsilon+|x|^{2}\right)^{-m}$ is a Fourier transform of a positive function in $L^{1}\left(\mathbf{R}^{n}\right)$. 
Proof. Combine

$$
\left(\varepsilon+|x|^{2}\right)^{-m}=\Gamma(m)^{-1} \int_{0}^{\infty} t^{m-1} e^{-\left(\varepsilon+|x|^{2}\right) t} d t, \quad m>0,
$$

with

$$
\exp \left(-|x|^{2} t\right)=(4 \pi t)^{-n / 2} \int_{\mathbf{R}^{n}} \exp \left(-|y|^{2} / 4 t\right) \exp (-\sqrt{-1} x \cdot y) d y,
$$

and note that the obtained double integral is absolutely convergent.

Proposition 5. For every $a \in A, \hat{P}_{a}$ does not vanish on $\mathfrak{M}(\mathbb{Q})$-the maximal ideal space of $\mathbb{Q}$.

Proof. (a) For the points $\left(t_{1}, \ldots, t_{r-1}\right) \in \mathfrak{M}(\mathbb{Q})$, integrating over $\mathbf{F}_{0}$ in the formula of Proposition 4(a), according to Lemma 1 (with $x_{0}=0$ ) we get

$$
\hat{P}_{a}\left(t_{1}, \ldots, t_{r-1}\right)=c \int_{\mathbf{R}^{o(r-1)}} \frac{\exp \left(\sqrt{-1} \operatorname{Re}\left(\bar{q}^{\prime} \cdot q\right)\right)}{\left(\varepsilon+|q|^{2}\right)^{d+1-\sigma}} d q,
$$

with

$$
c=2^{1-\sigma} \pi^{s-1 / 2} \varepsilon^{d / 2} c_{r, \mathbf{F}} \Gamma(d / 2-s+1 / 2) \Gamma(d / 2)^{-1},
$$

and this is positive by Lemma 2 and the Fourier inversion formula.

(b) For the points $\left(p^{\prime}, n\right) \in \mathfrak{M}(\mathbb{Q})$ with $p^{\prime} \in \mathbf{F}_{0} \backslash\{0\}, n=\left(n_{1}, \ldots, n_{r-1}\right) \in$ $\mathbf{N}^{r-1}$, we use the formula from Proposition 4(b) for $\hat{P}_{a}\left(p^{\prime}, n\right)$. Applying Lemma 1 to the integral over $\mathbf{F}_{0}$ there (with $Q=\varepsilon+t_{1}^{2}+\cdots+t_{r-1}^{2}, m=\sigma-1, x_{0}=p^{\prime}$, $k=d / 2$ ), then interchanging the order of integration from $d t d t_{1} \ldots d t_{r-1}$ to $d t_{1} \ldots d t_{r-1} d t$, making the change of variables $\left(\left|p^{\prime}\right| t_{1}^{2}, \ldots,\left|p^{\prime}\right| t_{r-1}^{2}\right)=$ $\left(x_{1}, \ldots, x_{r-1}\right)$, and finally applying (4.7), we obtain

$$
\hat{P}_{a}\left(p^{\prime}, n\right)=c \int_{0}^{\infty} e^{-\left|p^{\prime}\right| \varepsilon t / 2}\left((t+1)^{2}-1\right)^{d / 2-s} \prod_{l=1}^{r-1} g_{l}(t) d t,
$$

with

$$
c=2^{s(r-1)}\left(\varepsilon\left|p^{\prime}\right|\right)^{d / 2} \Gamma(d / 2)^{-1} \exp \left(-\left|p^{\prime}\right| \varepsilon / 2\right)
$$

and

$$
\oint_{l}(t)=\left(\left(n_{l}+s-1\right) !\right)^{-1} \int_{0}^{\infty} e^{-t x / 2}\left(d^{n_{l}} / d x^{n_{l}}\right)\left(x^{n_{l}+s-1} e^{-x}\right) d x
$$

Integrating by parts get

$$
\begin{aligned}
g_{l}(t) & =\left(\left(n_{l}+s-1\right) !\right)^{-1} \int_{0}^{\infty} e^{-t x / 2} x^{n_{l}+s-1} e^{-x} d x \cdot(t / 2)^{n_{l}} \\
& =(t / 2)^{n_{l}}(t / 2+1)^{-\left(n_{l}+s\right)} .
\end{aligned}
$$

Thus $\hat{P}_{a}\left(p^{\prime}, n\right)$ is positive.

REmark. For $M$ being the real hyperbolic space, i.e. for $\mathbf{F}=\mathbf{R}$, we have $N^{-}=\mathbf{R}^{d}, \mathfrak{M}\left(L^{1}\left(N^{-}\right)\right)=\hat{N}^{-}, P_{a}\left(X_{-\alpha}\right)=c_{d, \mathbf{R}} \varepsilon^{d / 2}\left(\varepsilon+|q|^{2}\right)^{-d}$ and, by Lemma 2, $\hat{P}_{a}>0$ on $\hat{N}^{-}$. 
6. Theorem on ideals in $L^{1}\left(N^{-}\right)$. Since the algebras $Q$ we consider here have the same qualitative properties as the one considered in [5], similar facts can be proved about them. In particular, the following statement about ideals in $L^{1}\left(N^{-}\right)$is a consequence of the Wiener property of $Q$ and existence of the approximate identity for $L^{1}\left(N^{-}\right)$in $Q$ (the dilations $\delta_{t}, t>0$, on $N^{-}$used in the construction of the approximate identity are given by $\left.\delta_{t}(q, p)=\left(t^{-1 / 2} q, t^{-1} p\right)\right)$.

Proposition 6. If 9 is a proper closed right ideal in $L^{1}\left(N^{-}\right)$, then there is a $\Phi$ in $\mathfrak{M}(\mathbb{Q})$ such that $\hat{F}(\Phi)=0$ for every $F \in \mathscr{G} \cap \mathbb{Q}$.

7. Proof of the Theorem [5]. The Theorem follows now from Proposition 6, for if we put

$$
g=\left\{f \in L^{1}\left(N^{-}\right): \lim _{N^{-} \ni n \rightarrow \infty} \varphi * f(n)=c_{0} \int_{N^{-}} f(n) d n\right\},
$$

with $\varphi \in L^{\infty}\left(N^{-}\right)$being the boundary value of the bounded harmonic function $u$ on $M$, then $P_{a_{0}} \in \mathcal{G} \cap \mathbb{Q}$ and $\hat{P}_{a_{0}} \neq 0$ on $\mathfrak{M}(\mathscr{Q})$, so $\mathcal{G}=L^{1}\left(N^{-}\right)$. Hence $P_{a} \in \mathcal{G}$ for every $a$ in $A$.

AdDED IN PROOF. Meanwhile Korányi [14] described the Gel'fand space, as well as the related Plancherel formula, for the commutative algebra $\mathcal{Q}$ of biradial functions in $L^{1}\left(N^{-}\right)$, i.e. the functions $F$ such that

$$
F(q, p)=f(|q|,|p|), \quad(q, p) \in N^{-},
$$

for some $f$ on $\mathbf{R}_{+} \times \mathbf{R}_{+}$, cf. $\$ 3$. His approach uses neither the classification of symmetric spaces nor the representations of nilpotent groups.

\section{REFERENCES}

1. P. Bernat et al., Représentations des groupes de Lie résolubles, Dunod, Paris, 1972.

2. D. Geller, Fourier analysis on the Heisenberg group, Proc. Nat. Acad. Sci. U.S.A. 74 (1977), $1328-1331$.

3. S. Helgason, Differential geometry and symmetric spaces, Academic Press, New York and London, 1962.

4. Application of the Radon transform to representations of semisimple Lie groups, Proc. Nat. Acad. Sci. U.S.A. 63 (1969), 643-647.

5. A. Hulanicki and F. Ricci, A Tauberian theorem and tangential convergence for bounded harmonic functions on balls in $C^{n}$, Invent. Math. 62 (1980), 325-331.

6. A. Kaplan and R. Putz, Boundary behavior of harmonic forms on a rank one symmetric space, Trans. Amer. Math. Soc. 231 (1977), 369-384.

7. A. Korányi, Boundary behavior of Poisson integrals on symmetric spaces, Trans. Amer. Math. Soc. 140 (1969), 393-409.

8. H. Leptin, Harmonische Analyse auf gewissen nilpotenten Lieschen Gruppen, Studia Math. 48 (1973), 201-205.

9. E. M. Stein and G. Weiss, Introduction to Fourier analysis on Euclidean spaces, Princeton Univ. Press, Princeton, N. J., 1971.

10. R. Takahashi, Quelques résultats sur l'analyse harmonique dans l'espace symétrique non compact de rang un du type exceptionnel, Analyse Harmonique sur les Groupes de Lie. II, Lecture Notes in Math., vol. 739, Springer-Verlag, Berlin and New York, 1979. 
11. G. Warner, Harmonic analysis on semi-simple Lie groups. I, Springer-Verlag, Berlin and New York, 1972.

12. G. N. Watson, A treatise on the theory of Bessel functions, 2nd ed., Cambridge Univ. Press, Cambridge, 1966.

13. D. P. Zhelobenko, Kompaktnye gruppy Li i ikh predstavleniya, Nauka, Moskva, 1970.

14. A. Korányi, Some applications of Gelfand pairs in classical analysis, Harmonic Analysis and Group Representations (C.I.M.E., Cortona, 1980), Liguori, Naples (to appear).

Institute of Mathematics, University of Wrockaw, Pl. Grunwaldzki 2/4, 50-384 Wrockaw, Poland 